\title{
Solid Sampling with a Diode Laser for Portable Ambient Mass Spectrometry
}

\section{Yung, Yeni P.}

2017-07-18

Yung, Y P , Wickramasinghe , R, Vaikkinen , A , Kauppila , T J , Veryovkin , I V \& Hanley , L 2017 , ' Solid Sampling with a Diode Laser for Portable Ambient Mass Spectrometry ' , Analytical Chemistry , vol. 89 , no. 14 , pp. 7297-7301 . https://doi.org/10.1021/acs.analchem.7b01745

http://hdl.handle.net/10138/232693

https://doi.org/10.1021/acs.analchem.7b01745

unspecified

publishedVersion

Downloaded from Helda, University of Helsinki institutional repository.

This is an electronic reprint of the original article.

This reprint may differ from the original in pagination and typographic detail.

Please cite the original version. 


\section{Solid Sampling with a Diode Laser for Portable Ambient Mass Spectrometry}

Yeni P. Yung, ${ }^{\dagger}$ Raveendra Wickramasinghe, ${ }^{\dagger}$ Anu Vaikkinen, ${ }^{\ddagger}$ Tiina J. Kauppila, ${ }^{\ddagger}$ Igor V. Veryovkin, ${ }^{\dagger}$ and Luke Hanley*,

${ }^{\dagger}$ Department of Chemistry, University of Illinois at Chicago, Chicago, Illinois 60607, United States

${ }^{\ddagger}$ Division of Pharmaceutical Chemistry and Technology, University of Helsinki, Helsinki, Finland

Supporting Information

ABSTRACT: A hand-held diode laser is implemented for solid sampling in portable ambient mass spectrometry (MS). Specifically, a pseudocontinuous wave batterypowered surgical laser diode is employed for portable laser diode thermal desorption (LDTD) at $940 \mathrm{~nm}$ and compared with nanosecond pulsed laser ablation at 2940 nm. Postionization is achieved in both cases using atmospheric pressure photoionization (APPI). The laser ablation atmospheric pressure photoionization (LAAPPI) and LDTD-APPI mass spectra of sage leaves (Salvia officinalis) using a field-deployable quadrupole ion trap MS display many similar ion peaks, as do the mass spectra of membrane grown biofilms of Pseudomonas aeruginosa. These results indicate that LDTD-APPI method should be useful for in-field sampling of plant and microbial communities, for example, by portable ambient MS. The feasibility of many portable MS applications is facilitated by the availability of relatively low cost, portable, battery-powered diode lasers. LDTD could also be coupled with plasma- or electrospray-based ionization for the analysis of a variety of solid samples.

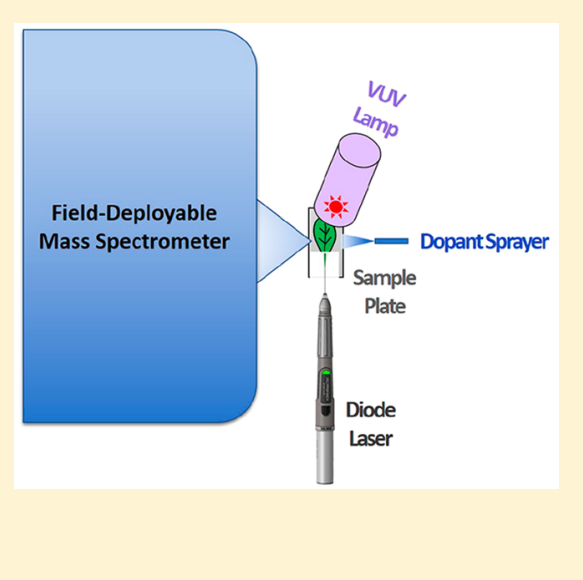

$\mathrm{T}$ here has been a rapid increase in the development of portable mass spectrometers (MS) and their application to the analysis of gaseous, liquid, and solid samples. ${ }^{1}$ Potential applications of portable MS include in-field sampling of native plant $^{2,3}$ or microbial communities. ${ }^{4-6}$ For example, portable MS could be used for quality control in herbal supplements ${ }^{7}$ or to identify microbial colonies on medical devices for the purpose of disease prevention. ${ }^{8}$

Along with other classes of ion sources used for $\mathrm{MS},{ }^{9}$ ambient or atmospheric pressure sources have been investigated for use in portable MS due to the convenience imparted by direct sampling without the extraction, pyrolysis, and/or desiccation required by traditional MS sample preparation methods. ${ }^{10}$ Desorption electrospray ionization $(\mathrm{DESI})^{11-13}$ and plasma discharge-based ion sources ${ }^{14-16}$ are both wellestablished for direct sampling of solids in portable ambient MS. However, such ion sources can suffer from fluctuations in the efficiency with which analytes are extracted/volatilized then ionized. For example, the efficiency with which plasma-based sources will detect analytes in a solid sample will depend on the specific plasma conditions and source-to-sample distance as well as sample volatility and thermal stability. ${ }^{14-16}$

Laser sampling induces desorption/ablation of analyte from the surface of a solid sample and can additionally permit MS imaging at relatively high lateral resolution. ${ }^{9}$ Laser desorption/ ablation is also well established for sampling at atmospheric pressure, especially using ultraviolet or infrared lasers with pulse lengths ranging from $\sim 10 \mathrm{~ns}$ to $<100 \mathrm{fs}$. ${ }^{9,17-20}$ However, such lasers are usually too expensive, delicate, and complicated for routine application in portable ambient MS, especially when compared with DESI, paper spray, or other plasma-based methods. A potential portable solution can be found in laser diode thermal desorption (LDTD), ${ }^{21}$ which uses continuous wave $(\mathrm{CW})$ or pseudo-CW near-IR diode lasers to volatilize solid samples.

This technical note demonstrates the implementation of a hand-held diode laser for solid sampling in portable ambient MS. A battery-powered surgical laser diode is employed for portable LDTD at $940 \mathrm{~nm}$ and compared with laser ablation by a laboratory-based nanosecond pulsed laser operating at 2940 nm. Postionization is achieved using atmospheric pressure photoionization (APPI). ${ }^{18}$ Experiments that used the $940 \mathrm{~nm}$, portable laser diode are referred to as LDTD-APPI, while those that used the pulsed $2940 \mathrm{~nm}$ laboratory laser are referred to as LAAPPI, for consistency with published nomenclature. ${ }^{18,21}$ These experiments compare the analysis of intact plant leaves and bacterial biofilms by LDTD-APPI with the established method of LAAPPI, ${ }^{18,21}$ thereby demonstrating the feasibility of LDTD-APPI for in-field sampling of native plant or microbial communities. A preliminary version of the LDTD-APPI source was previously reported. ${ }^{22}$

Received: May 9, 2017

Accepted: June 20, 2017

Published: June 20, 2017 


\section{EXPERIMENTAL DETAILS}

Ion Source and Ambient MS. The home-built LDTDAPPI source shown in Figure 1 is based on an APPI source ${ }^{23}$

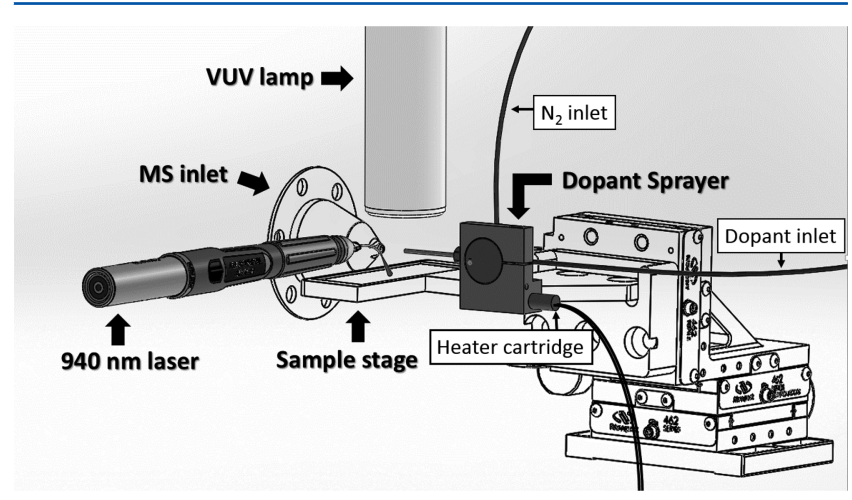

Figure 1. Schematic diagram of the laser diode thermal desorption atmospheric pressure photoionization (LDTD-APPI) source with a battery-powered, portable, pseudo-CW $940 \mathrm{~nm}$ diode laser.

that consists of a vacuum ultraviolet (VUV) photoionization lamp and dopant gas nebulizer, to which a laser has been added for desorption/ablation. A radio frequency-powered krypton gas discharge VUV lamp (KrLM-LQD12, Resonance Ltd., Barrie, Ont., Canada) that outputs at $10.0 / 10.6 \mathrm{eV}$ is used for photoionization. Dopant vapor is used in most of the experiments presented here as it has been previously shown to improve ionization efficiency in APPI through charge transfer and proton exchange. ${ }^{24,25}$ The nebulizer for dopant delivery was inspired by a published design ${ }^{23}$ and consists of a stainless steel tee-junction (VICI Valco, Houston, TX) that is fitted to a stainless steel heater block adapter machined inhouse to incorporate a heater cartridge (Watlow FIREROD, Ash Equipment Co., Batavia, IL). The tee-junction has two inputs for dopant delivery from a syringe pump and nitrogen gas delivery, and an output to an effluent capillary tip that delivers the heated dopant vapor close to the intersection of the laser desorption/ablation spot on the sample, the VUV ionization region, and the atmospheric pressure aperture of the MS

This LDTD-APPI source uses a CW diode laser operating at $940 \mathrm{~nm}$ for sample desorption (iLase, Biolase Tech, Irvine, CA). This hand-held laser (100 g, $21 \mathrm{~cm}$ long) is designed for dental surgical applications, is equipped with $1 \mathrm{~h}$ lifetime rechargeable batteries, has a $3 \mathrm{~W}$ peak power optical output, and is typically operated in a pseudo-CW mode $(0.1 \mathrm{~ms}$ on $/ 0.2$ $\mathrm{ms}$ off). The laser beam is guided to the ablation area via a disposable, $400 \mu \mathrm{m}$ fiber optic tip that can be bent to accommodate spatially constricted samples. A diode laser output of $\sim 700 \mathrm{~mW}$ output (power density $\sim 140 \mathrm{~W} / \mathrm{cm}^{2}$ ) was generally used to collect the LDTD-APPI mass spectra.

The LAAPPI source follows a previously reported configuration, ${ }^{18}$ but with the same VUV lamp and dopant vapor delivery scheme, as shown in Figure 1 and described above. LAAPPI employs a $\sim 7 \mathrm{~ns}, 20 \mathrm{~Hz}$ pulsed, laboratory laser operating at $2940 \mathrm{~nm}$ composed of an optical parametric oscillator pumped by a Nd:YAG laser (Opolette 2940, Opotek, Carlsbad, CA). The laser output is focused via a biconvex $\mathrm{CaF}_{2}$ lens into a $450 \mu \mathrm{m}$ core $\mathrm{GeO}_{2}$ fiber patch (Infrared Fiber Systems, Silver Springs, MD) and the fiber output beam is collimated and focused through two plano-convex $\mathrm{CaF}_{2}$ lenses directly onto the sample. The output from the fiber optic in
LAAPPI was generally $\sim 2 \mathrm{~mJ} /$ pulse (power density $\sim 25 \mathrm{~W}$ / $\mathrm{cm}^{2}$ ).

The mass spectrometric analyses were performed using a field-deployable 3D ion trap (MT Explorer 50, MassTech, Columbia, MD). ${ }^{26}$ The inlet cone was set to $120{ }^{\circ} \mathrm{C}$ and the capillary voltage at $10 \mathrm{VDC}$, with automatic gain control enabled with target value set at 100,000. A high transmission wire grid was also mounted on the output end of the VUV lamp (not shown in Figure 1) and connected to both the sample plate and the capillary (including the aperture cone to the mass spectrometer) so that all three sat at the same voltage during analysis. The resultant electric field-free region in the vicinity of the MS aperture was an attempt to minimize signal losses due to possible ion deflection away from the aperture caused by sample charging effects and/or an electric field gradient. Minimum and maximum injection times for individual mass scans were 1 and $1000 \mathrm{~ms}$, respectively, for the sage analysis. Biofilm analyses used a fixed injection time of $100 \mathrm{~ms}$. Spectra were averaged over one min of individual scans for sage and two min for biofilms.

Chemicals, Samples, and Replicates. Anisole (99.7\%) and toluene $(\geq 99.9 \%)$ were used as purchased (Sigma-Aldrich, St. Louis, MO). 4-Amino-5-chloro-2-methoxybenzoic acid $\left(\mathrm{C}_{8} \mathrm{H}_{8} \mathrm{ClNO}_{3}, 97 \%\right)$ (Sigma-Aldrich, St. Louis, $\mathrm{MO}$ ) was dissolved in spectroscopic grade methanol to a concentration of $10 \mathrm{mg} / \mathrm{mL}$ then deposited onto chitosan-alginate polyelectrolyte multilayers adsorbed onto gold coated silicon wafers, prepared as described previously. ${ }^{27}$ LDTD-APPI and LAAPPI analyses of the resultant standard samples were replicated at least four times while rastering the sample with respect to the laser spot at a rate of $0.2 \mathrm{~mm} / \mathrm{s}$.

Organic sage leaves (Salvia officinalis) were purchased from a local supermarket and stored at $\sim 4{ }^{\circ} \mathrm{C}$ prior to analysis. The leaves were washed with deionized, distilled water to remove dirt, blotted dry, and then mounted onto a glass slide using double-sided copper tape with the abaxial side facing up. LDTD-APPI and LAAPPI analyses were replicated on at least five replicates rastering at $0.3 \mathrm{~mm} / \mathrm{s}$ with $5 \mu \mathrm{L} / \mathrm{min}$ anisole dopant and $\sim 80 \mathrm{~mL} / \mathrm{min}$ nitrogen flow rates.

A total of $10 \mu \mathrm{L}$ of Pseudomonas aeruginosa clinical isolate overnight culture at $0.1 \mathrm{OD}_{600}$ were inoculated onto a polycarbonate membrane placed on top of nutrient-defined Clostridium Staphylococcus Pseudomonas agar plate and grown for 3 days. The resultant biofilms were exposed to $0.1 \%$ trifluoroacetic acid for $1 \mathrm{~h}$ for sterilization, transferred onto a stainless steel plate, and frozen to remove the membrane prior to analysis. LDTD-APPI and LAAPPI analyses were performed on at least seven distinct biofilm samples, rastering at $0.03 \mathrm{~mm} /$ $\mathrm{s}$ with $0.2 \mu \mathrm{L} / \mathrm{min}$ toluene dopant flow rate, and $\sim 50 \mathrm{~mL} / \mathrm{min}$ nitrogen flow rate.

\section{RESULTS AND DISCUSSION}

LAAPPI (2940 nm, $\sim 7 \mathrm{~ns}$ pulsed) and LDTD-APPI $(940 \mathrm{~nm}$, pseudo-CW) mass spectra of the 4-amino-5-chloro-2-methoxybenzoic acid (MACA, 201.02 Da) standard both show intense $\mathrm{MH}^{+}$ions in Figure 2, although LDTD-APPI displayed more fragmentation. The dopant vapor was not required in either case and was therefore not used. Both LAAPPI and LDTDAPPI displayed the 3:1 peak intensity ratio at $\mathrm{m} / z 201.6$ and 203.6 attributed to the $\mathrm{MH}^{+}$ion that is characteristic of the ${ }^{35} \mathrm{Cl} /{ }^{37} \mathrm{Cl}$ isotopic ratio expected for MACA. Tandem MS experiments detected ions resulting from losses of $\mathrm{ClCOOH}$ and $\mathrm{ClCH}_{2} \mathrm{COOH}$ from $\mathrm{MH}^{+}$, confirming this assignment 


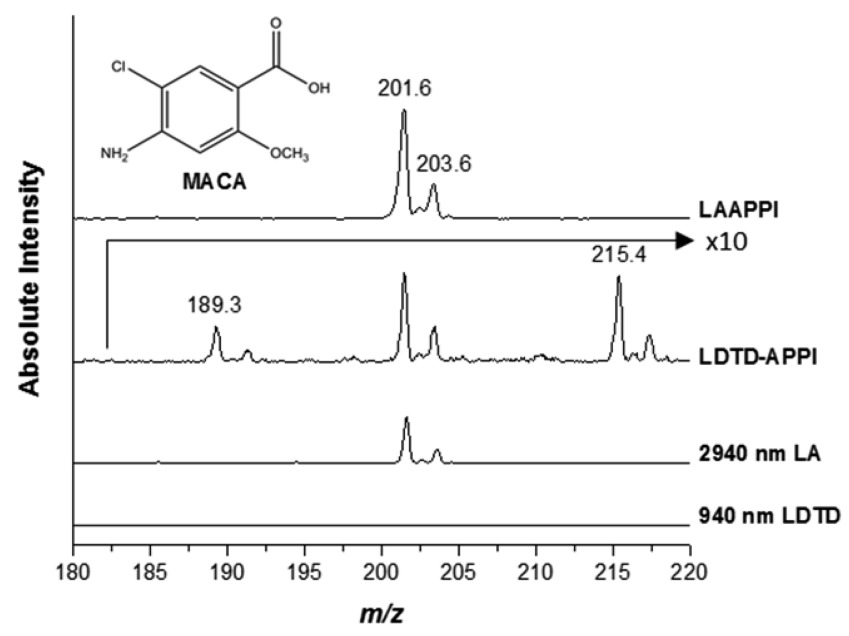

Figure 2. Mass spectra of the MACA standard (inset shows chemical structure) analyzed by (from top to bottom) $2940 \mathrm{~nm}$ laser ablation atmospheric pressure photoionization (LAAPPI), LDTD-APPI, 2940 nm laser ablation (without VUV lamp), and $940 \mathrm{~nm} \mathrm{LDTD} \mathrm{(without}$ VUV).

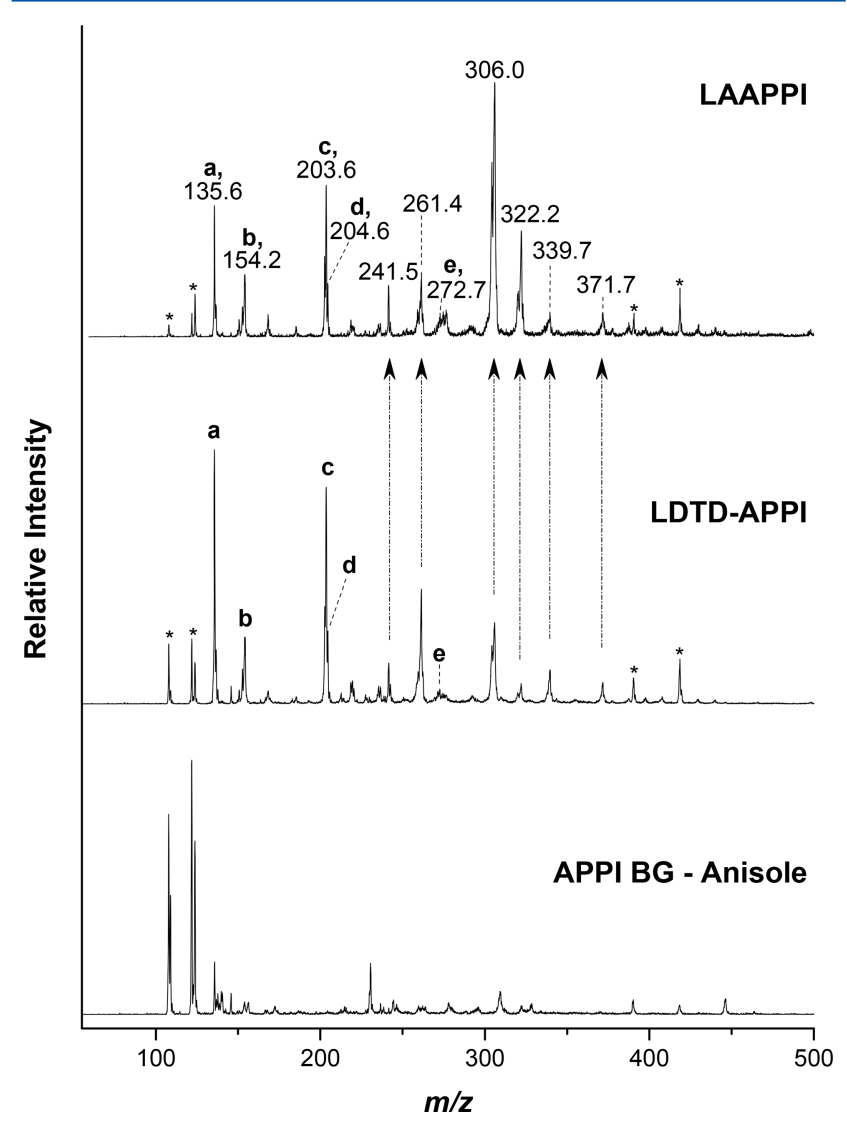

Figure 3. Representative analyses of sage leaves by (top) LAAPPI and (middle) LDTD-APPI with anisole dopant. Anisole dopant background in APPI (bottom). The letters "(a)" to "(e)" annotate peaks observed by both LAAPPI and LDTD-APPI for whom with tentative assignments were possible based on phytochemicals previous detected by LAAPPI (see Supporting Information). The arrows indicate other peaks observed in the LDTD-APPI mass spectrum that are labeled in the LAAPPI mass spectrum. * indicates background peaks.

(data not shown). Direct laser desorption ionization (without VUV lamp) of MACA was also observed for the $2940 \mathrm{~nm}$ laser, but not for $940 \mathrm{~nm}$ LDTD. The VUV lamp generated a signal
Table 1. Peaks Observed in LAAPPI- and LDTD-APPI-MS Spectra of Sage Leaves with Anisole as the Dopant ${ }^{a}$

\begin{tabular}{|c|c|c|}
\hline LAAPPI $( \pm m / z 0.1)$ & LDTD-APPI $( \pm m / z 0.1)$ & LAAPPI $(m / z)$ from ref 28 \\
\hline (a) 135.6 & (a) $135.6^{c}$ & 135.1 \\
\hline 136.6 & & 136.1 \\
\hline (b) 154.2 & (b) 154.2 & 154.1 \\
\hline (c) 203.6 & (c) 203.6 & 203.2 \\
\hline \multirow[t]{2}{*}{ (d) 204.6} & (d) 204.5 & 204.2 \\
\hline & 219.5 & 219.2 \\
\hline 241.5 & 241.4 & \\
\hline 261.4 & 261.4 & \\
\hline \multirow[t]{2}{*}{ (e) $272.7^{b}$} & (e) 272.6 & 272.2 \\
\hline & 304.3 & \\
\hline 306.0 & 306.0 & \\
\hline 320.3 & 320.2 & \\
\hline 322.2 & 322.1 & \\
\hline \multirow[t]{2}{*}{339.7} & 339.7 & \\
\hline & 340.7 & \\
\hline 371.7 & 371.7 & \\
\hline
\end{tabular}

${ }^{a}$ Peaks were included only if they were observed in $\geq 50 \%$ of the samples with $>5$ signal-to-noise $(\mathrm{S} / \mathrm{N})$ when compared against the anisole background. The letters "(a)" to "(e)" annotate the same peaks so noted in Figure 3, while italicized peaks in LDTD-APPI are those that also appeared with toluene dopant. ${ }^{b}$ Denotes low $\mathrm{S} / \mathrm{N}$ peak. ${ }^{c}$ LDTD-APPI peaks that appear with both anisole and toluene dopant are italicized.

via postionization for LDTD-APPI and enhanced the signal with LAAPPI (see Figure 2). Also observed in the LDTD-APPI mass spectra of Figure 2 were peaks at $m / z 215.4$, $[M+14]^{+}$ and $m / z 189.3,[\mathrm{M}-12]^{+}$. The characteristic chlorine isotopic distributions of these two peaks indicate they derive from ion molecule reactions followed by fragmentation and/or rearrangement of precursor ions.

LAAPPI was previously used to analyze phytochemicals of sage leaves, ${ }^{28}$ so this analysis was repeated here as a sort of "real life" standard and compared in Figure 3 with an analysis of sage leaves by LDTD-APPI. Both anisole and toluene were used as dopants given that their low ionization energies (8.20 and 8.83 $\mathrm{eV}$, respectively) are well below the photon energy of the VUV source $(10.0 / 10.6 \mathrm{eV})$. However, Figure 3 displays only the results with anisole due to its lower background.

The LAAPPI and LDTD-APPI mass spectra of sage leaves shown in Figure 3 appear rather similar to a cursor visual examination. Table 1 outlines the 12 common peaks that were observed from sage leaves by both methods, as well as the three additional peaks observed exclusively by only one of the two methods, obtained by averaging all five replicates. The letters "(a)" to "(e)" annotate peaks observed by both LAAPPI and LDTD-APPI (see Table 1) for whom tentative assignments were additionally made based on phytochemicals previously reported from sage leaves or extracts thereof (see Supporting Information). ${ }^{28,29}$

However, it is important to note that all the peak assignments are only tentative and are based on prior GCMS, LC-MS, and LAAPPI studies. ${ }^{28,29}$ The \pm 0.5 Da mass error of the field deployable ion trap used here limits the peak assignment accuracy. Furthermore, attempts to collect tandem MS data directly from the sage leaves were unsuccessful, presumably due to the low sensitivity of the ion trap mass analyzer. Peaks that were not observed here, but were observed previously $^{28}$ by LAAPPI include $m / z$ 169.1, 286.2, 300.2, 315.1, 316.2, 331.2, 332.2, and 346.2. Differences in the peaks 


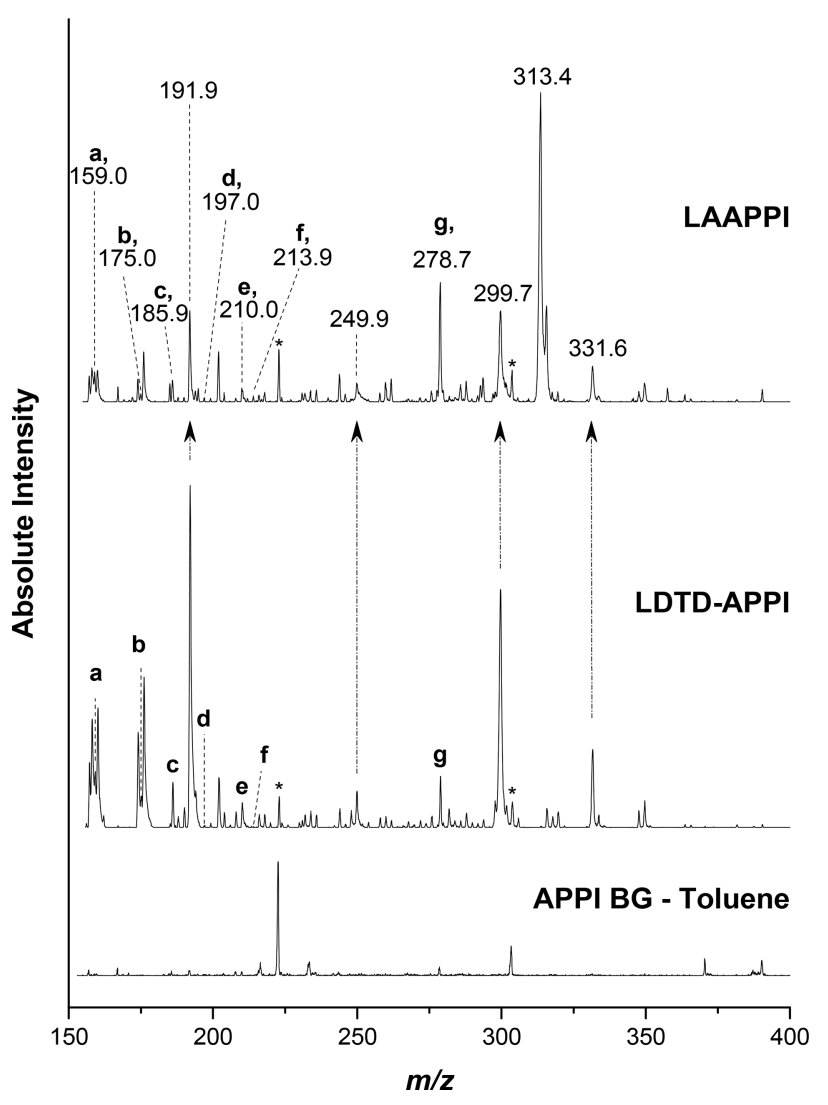

Figure 4. Representative analyses of Pseudomonas aeruginosa membrane-grown biofilms by (top) LAAPPI and (middle) LDTDAPPI with toluene. Toluene dopant background in APPI (bottom). The peaks annotated with "(a)" to "(g)" annotated in the figure correspond to peaks observed in both LAAPPI and LDTD-APPI that have been tentatively assigned to known metabolites reported in literature (see Supporting Information). ${ }^{4,30-33}$ The arrows indicate other peaks observed in the LDTD-APPI mass spectrum that are labeled in the LAAPPI mass spectrum. * indicates background peaks.

observed here and previously by LAAPPI ${ }^{28}$ might be attributed to the use of different mass analyzers, MS source conditions, and sage leaf growth conditions, storage conditions, and/or genetics.

The LAAPPI and LDTD-APPI mass spectra shown in Figure 4 of membrane-grown Pseudomonas aeruginosa biofilms are also quite similar by visual inspection. Table 2 lists 21 peaks observed by both methods and an additional two peaks observed exclusively by only one method, obtained by averaging all seven replicates. Of these 21 common peaks, those annotated with the lower case letters "(a)" to " $(\mathrm{g})$ " in Figure 4 and Table 2 were those that could additionally be tentatively assigned to metabolites previously reported for $P$. aeruginosa cultures by various MS strategies (see Supporting Information). ${ }^{4,30-33}$ Overall, peaks were observed whose $\mathrm{m} / \mathrm{z}$ values can be attributed to phenazines, homoserine lactones (HSL), Pseudomonas quinolone signal (PQS) and hydroxyalkyl-quinolones (HAQs) that were previously detected by various mass spectrometric methods and are thought to participate in inter- and intraspecies cellular communication (see Supporting Information). ${ }^{34}$ However, the same caveats regarding peak assignments stated above for the sage leaf MS also apply to the biofilms.
Table 2. Peaks Observed from P. aeruginosa Biofilms by LAAPPI and LDTD-APPI ${ }^{a}$

\begin{tabular}{|cc} 
LAAPPI $( \pm m / z$ 0.1) & LDTD-APPI $( \pm m / z 7.1$ \\
157.1 & 158.1 \\
158.0 & (a) 159.1 \\
(a) 159.0 & 160.0 \\
160.0 & 174.0 \\
173.9 & (b) 175.1 \\
(b) 175.0 & 176.0 \\
176.0 & 185.1 \\
185.0 & (c) $186.0^{b}$ \\
(c) 185.9 & 192.0 \\
191.9 & (d) 197.0 \\
(d) 197.0 & 199.2 \\
199.1 & 202.0 \\
201.9 & $203.9^{b}$ \\
& (e) 210.1 \\
(e) 210.0 & (f) 214.0 \\
(f) 213.9 & 230.9 \\
230.8 & 249.9 \\
249.9 & (g) 278.8 \\
(g) 278.7 & 287.8 \\
$287.7^{b}$ & 299.5 \\
299.7 & \\
313.4 & 331.5 \\
331.6 &
\end{tabular}

${ }^{a}$ Peaks were included in Table 2 only if they were observed in $\geq 50 \%$ of the samples with $>5$ signal-to-noise $(\mathrm{S} / \mathrm{N})$ when compared against the toluene background. The letters "(a)" to "(g)" annotate the same peaks so noted in Figure $4 .{ }^{b}$ Denotes low $\mathrm{S} / \mathrm{N}$ peak.

\section{CONCLUSIONS}

The LDTD-APPI method should be useful for solid sampling in portable ambient MS in in-field sampling of native plant ${ }^{2,3}$ or microbial communities ${ }^{4-6,8}$ as well as other potential applications. The feasibility of many portable MS applications is facilitated by the availability of low cost, portable, batterypowered diode lasers. Furthermore, the fact that LDTD-APPI gives similar results to LAAPPI, at least for sage leaves and $P$. aeruginosa biofilms, argues for the use of LDTD-APPI in cases where portability and lower cost are desirable.

The similarity of spectra is also fundamentally interesting given that sample volatilization in LDTD presumably occurs via thermal desorption, ${ }^{21}$ whereas LAAPPI is thought to proceed via explosive evaporation of water that ejects a hydrated sample into the gas phase. ${ }^{18,35}$ The separation of the desorption/ ablation and ionization steps indicates that photoionization of similar volatilized species is occurring in both LDTD-APPI and LAAPPI.

LDTD-APPI is expected to enhance sample volatilization via thermal heating, when compared with desorption atmospheric pressure photoionization (DAPPI), which proceeds via a hot solvent jet impinging upon the sample. ${ }^{36}$ LDTD might also be effective when coupled to plasma-based ion sources that are used for solid sampling, ${ }^{15,16,37}$ since LDTD might be used to reduce fluctuations in volatilization efficiency that arise from sample heating by the plasma, which will vary with specific plasma conditions and source-to-sample distance. ${ }^{14,37}$ Plasma power has a direct relationship with sample surface heating, but also affects the extent of molecular fragmentation ${ }^{14}$ as well as ionization efficiency. It is speculated that the addition of LDTD might be more reproducible than plasma-based ionization alone 
by enhancing sample volatilization. Finally, LDTD could readily be coupled to electrospray ionization for LDTD-ESI, a portable method analogous to laser ablation electrospray ionization (LAESI). ${ }^{17}$

\section{ASSOCIATED CONTENT}

\section{S Supporting Information}

The Supporting Information is available free of charge on the ACS Publications website at DOI: 10.1021/acs.analchem.7b01745.

Tentative peak assignments for the mass spectra of sage leaves and $P$. aeruginosa biofilms (PDF).

\section{AUTHOR INFORMATION}

\section{Corresponding Author}

*E-mail: lhanley@uic.edu. Phone: +1-312-996-0945.

\section{ORCID}

Luke Hanley: 0000-0001-7856-1869

Notes

The authors declare no competing financial interest.

\section{ACKNOWLEDGMENTS}

The authors acknowledge Ross P. Carlson of Montana State University for providing the bacterial cultures and advice in growing the biofilms, and Michael Colvard of UIC for the loan of the portable laser. This work was funded by the National Institute of Biomedical Imaging and Bioengineering under grant 1U01EB019416, the U.S. Air Force SGR Directorate of Modernization under Contract FA7014-09-2-0003-T4T, the Academy of Finland (Grant \# 218150, 255559, and 275089) and the CHEMSEM Doctoral School (Helsinki).

\section{REFERENCES}

(1) Snyder, D. T.; Pulliam, C. J.; Ouyang, Z.; Cooks, R. G. Anal. Chem. 2016, 88, 2-29.

(2) Lancaster, C.; Espinoza, E. Rapid Commun. Mass Spectrom. 2012, 26, 1147-1156.

(3) Pavarini, D. P.; da Silva, D. B.; Carollo, C. A.; Portella, A. P. F.; Latansio-Aidar, S. R.; Cavalin, P. O.; Oliveira, V. C.; Rosado, B. H. P.; Aidar, M. P. M.; Bolzani, V. S.; Lopes, N. P.; Joly, C. A. J. Mass Spectrom. 2012, 47, 1482-1485.

(4) Moree, W. J.; Phelan, V. V.; Wu, C.-H.; Bandeira, N.; Cornett, D. S.; Duggan, B. M.; Dorrestein, P. C. Proc. Natl. Acad. Sci. U. S. A. 2012, 109, 13811-13816.

(5) Leefmann, T.; Heim, C.; Siljeström, S.; Blumenberg, M.; Sjövall, P.; Thiel, V. Rapid Commun. Mass Spectrom. 2013, 27, 565-581.

(6) Sumner, D. Y.; Hawes, I.; Mackey, T. J.; Jungblut, A. D.; Doran, P. T. Geology 2015, 43, 887-890.

(7) García-Cañas, V.; Simó, C.; Herrero, M.; Ibáñez, E.; Cifuentes, A. Anal. Chem. 2012, 84, 10150-10159.

(8) Vertes, A.; Hitchins, V.; Phillips, K. S. Anal. Chem. 2012, 84, $3858-3866$.

(9) Bhardwaj, C.; Hanley, L. Nat. Prod. Rep. 2014, 31, 756-767.

(10) Ifa, D. R.; Wu, C.; Ouyang, Z.; Cooks, R. G. Analyst 2010, 135, 669-681.

(11) Mulligan, C. C.; Talaty, N.; Cooks, R. G. Chem. Commun. 2006, $1709-1711$.

(12) Keil, A.; Talaty, N.; Janfelt, C.; Noll, R. J.; Gao, L.; Ouyang, Z.; Cooks, R. G. Anal. Chem. 2007, 79, 7734-7739.

(13) Hendricks, P. I.; Dalgleish, J. K.; Shelley, J. T.; Kirleis, M. A.; McNicholas, M. T.; Li, L.; Chen, T.-C.; Chen, C.-H.; Duncan, J. S.; Boudreau, F.; Noll, R. J.; Denton, J. P.; Roach, T. A.; Ouyang, Z.; Cooks, R. G. Anal. Chem. 2014, 86, 2900-2908.
(14) Salter, T. L. R.; Bunch, J.; Gilmore, I. S. Anal. Chem. 2014, 86, 9264-9270.

(15) Wiley, J. S.; Shelley, J. T.; Cooks, R. G. Anal. Chem. 2013, 85, $6545-6552$.

(16) Albert, A.; Shelley, J.; Engelhard, C. Anal. Bioanal. Chem. 2014, 406, 6111-6127.

(17) Nemes, P.; Barton, A. A.; Li, Y.; Vertes, A. Anal. Chem. 2008, 80, $4575-4582$.

(18) Vaikkinen, A.; Shrestha, B.; Kauppila, T. J.; Vertes, A.; Kostiainen, R. Anal. Chem. 2012, 84, 1630-1636.

(19) Flanigan, P.; Levis, R. Annu. Rev. Anal. Chem. 2014, 7, 229-256.

(20) Cui, Y.; Veryovkin, I. V.; Majeski, M. W.; Cavazos, D. R.; Hanley, L. Anal. Chem. 2015, 87, 367-371.

(21) Wu, J.; Hughes, C. S.; Picard, P.; Letarte, S.; Gaudreault, M.; Lévesque, J.-F.; Nicoll-Griffith, D. A.; Bateman, K. P. Anal. Chem. 2007, 79, 4657-4665.

(22) Yung, Y. P.; Veryovkin, I. V.; Cui, Y.; Hanley, L. Proc. 63rd ASMS Conf. on Mass Spectrom. and Allied Topics, 31 May - 4 June, St. Louis, Misssouri, American Society for Mass Spectrometry, 2015; WP430.

(23) Kauppila, T. J.; Östman, P.; Marttila, S.; Ketola, R. A.; Kotiaho, T.; Franssila, S.; Kostiainen, R. Anal. Chem. 2004, 76, 6797-6801.

(24) Robb, D. B.; Covey, T. R.; Bruins, A. P. Anal. Chem. 2000, 72, $3653-3659$.

(25) Kauppila, T. J.; Kuuranne, T.; Meurer, E. C.; Eberlin, M. N.; Kotiaho, T.; Kostiainen, R. Anal. Chem. 2002, 74, 5470-5479.

(26) Misharin, A.; Novoselov, K.; Laiko, V.; Doroshenko, V. M. Anal. Chem. 2012, 84, 10105-10112.

(27) Blaze, M. T.; Takahashi, L. K.; Zhou, J.; Ahmed, M.; Gasper, G. L.; Pleticha, F. D.; Hanley, L. Anal. Chem. 2011, 83, 4962-4969.

(28) Vaikkinen, A.; Shrestha, B.; Koivisto, J.; Kostiainen, R.; Vertes, A.; Kauppila, T. J. Rapid Commun. Mass Spectrom. 2014, 28, 24902496.

(29) Santos-Gomes, P. C.; Fernandes-Ferreira, M. J. Agric. Food Chem. 2001, 49, 2908-2916.

(30) Lépine, F.; Milot, S.; Déziel, E.; He, J.; Rahme, L. G. J. Am. Soc. Mass Spectrom. 2004, 15, 862-869.

(31) Lanni, E. J.; Masyuko, R. N.; Driscoll, C. M.; Dunham, S. J. B.; Shrout, J. D.; Bohn, P. W.; Sweedler, J. V. Anal. Chem. 2014, 86, 10885-10891.

(32) Dunham, S. J. B.; Comi, T. J.; Ko, K.; Li, B.; Baig, N. F.; Morales-Soto, N.; Shrout, J. D.; Bohn, P. W.; Sweedler, J. V. Biointerphases 2016, 11, 02A325.

(33) Cui, Y.; Frey, R. L.; Ferry, J. L.; Ferguson, P. L. Rapid Commun. Mass Spectrom. 2009, 23, 1212-1220.

(34) De Kievit, T. R. Environ. Microbiol. 2009, 11, 279-288.

(35) Chen, Z.; Vertes, A. Phys. Rev. E 2008, 77, 036316.

(36) Luosujärvi, L.; Arvola, V.; Haapala, M.; Pol, J.; Saarela, V.; Franssila, S.; Kotiaho, T.; Kostiainen, R.; Kauppila, T. J. Anal. Chem. 2008, 80, 7460-7466.

(37) McKay, K.; Salter, T.; Bowfield, A.; Walsh, J.; Gilmore, I.; Bradley, J. J. Am. Soc. Mass Spectrom. 2014, 25, 1528-1537. 